\title{
An Evaluation of Motivational Interviewing for Increasing Hearing Aid Use: A Pilot Study
}

DOI: $10.3766 /$ jaaa. 16184

\author{
Jorunn Solheim* \\ Caryl Gay* \\ Anners Lerdal*† \\ Louise Hickson $\neq$ \\ Kari J. Kvaerner§
}

\begin{abstract}
Background: Motivational interviewing (MI) has been used in consultation settings to motivate hearing aid users to increase hearing aid usage. However, the effect of $\mathrm{MI}$ on those who use their hearing aids only rarely or not at all has not been explored.

Purpose: The aims of this pilot study were to evaluate the effect of MI counseling with elderly hearing aid recipients found to have low hearing aid use at a six-month follow-up appointment and to describe clients' subjective assessments of their perceived need for hearing aids three months after Ml counseling.
\end{abstract}

Research Design: The study had a within-subjects pretest-posttest design.

Study Sample: Forty seven hearing aid recipients who had used their new hearing aids, an average of $<90 \mathrm{~min} /$ day, were recruited at a follow-up appointment six months after hearing aid fitting.

Intervention: Thirty minutes of $\mathrm{Ml}$ counseling was provided at the six-month follow-up appointment. If needed, hearing aid adjustments and technical support were also provided.

Data Collection and Analysis: The effect of MI counseling in combination with adjustments and technical support was assessed in relation to datalogged hearing aid use, which was assessed immediately before (at the six-month follow-up) and three months after (at the nine-month follow-up) the intervention. Hearing aid experiences were also assessed three months after MI.

Results: Thirty seven participants (79\%) returned for the nine-month follow-up visit and had modest but significant increases in datalogged hearing aid use in the three months following MI counseling. Of the 37 participants who returned, $51 \%$ had increased their hearing aid use to at least $2 \mathrm{~h}$ /day after the Ml counseling. Most of the 37 participants who attended the nine-month follow-up reported increased need for $(59 \%)$ or increased benefit and contentment with (57\%) their hearing aid three months after $\mathrm{Ml}$; these participants also had significantly higher datalogged hearing aid use following MI.

Conclusions: These findings suggest that follow-up appointments using $\mathrm{Ml}$ counseling in conjunction with technical support may be useful for increasing hearing aid usage among low-users, and a randomized controlled trial is warranted.

Key Words: elderly, hearing aid, hearing impaired, motivational interview

Abbreviations: $\mathrm{MI}=$ motivational interviewing; $\mathrm{MISC}=$ motivational interviewing with standard care; OARS = Open questions, Affirmation, Reflective listening, Summary reflections; PTA = pure-tone average audiometric threshold; $\mathrm{RCT}=$ randomized controlled trial; $\mathrm{SC}=$ standard care; $\mathrm{SD}=$ standard deviation

*ENT Department, Lovisenberg Diakonale Hospital, Oslo, Norway; †Department of Health and Society, Faculty of Medicine, University of Oslo, Oslo, Norway; $¥$ School of Health and Rehabilitation Sciences, University of Queensland, Brisbane, Australia; §C3 Centre for Connected Care, Oslo University Hospital, Oslo, Norway

Corresponding author: Jorunn Solheim, Lovisenberg Diakonale Hospital, 0456 Oslo, Norway; Email: jorunn.solheim@Ids.no

Funding for this research was provided by a grant from the Norwegian Foundation for Health and Rehabilitation (ExtraStiftelsen), awarded to the first author. The Norwegian Association of the Hearing Impaired (HLF) stood as applicant organization. 


\section{BACKGROUND}

$\mathrm{P}$ rior studies have raised concern about the considerable number of hearing aids not being used (Lupsakko et al, 2005; Gimsing, 2008; Maeda et al, 2016). It is estimated that up to $24 \%$ of people with hearing aids seldom ( $\leq 1 \mathrm{~h} /$ day) or never use them (Stark and Hickson, 2004; Vuorialho et al, 2006; KaplanNeeman et al, 2012; Solheim et al, 2012). Commonly reported reasons for nonuse of hearing aids include disappointment in their level of effectiveness (particularly in noisy situations), low motivation because of little perceived need for a hearing aid, difficulty handling the small parts, and social stigma (Gussekloo et al, 2003; Solheim, 2011; Hickson et al, 2014; Meyer, Hickson, Lovelock, et al, 2014; Chang et al, 2016; Cherko et al, 2016). These factors are considered to be of substantial importance to treatment adherence and subsequent satisfaction with hearing aids. In addition, follow-up support has been recommended to identify low-users and to ensure that frequently experienced barriers do not result in loss of motivation (Gianopoulos et al, 2002; Solheim et al, 2012).

Motivational interviewing (MI) has been explored as an approach to help people learn new behavioral skills and motivate for change. The primary goal of MI is to enhance the client's intrinsic motivation. The method emphasizes the client's autonomy and is based on the client's own experiences. This person-centered counseling style is collaborative and involves a partnership between the provider and client to address ambivalence about behavior change. It includes four stages: engaging (the client), focusing (on the desired behavior change), evoking (ideas, commitments, values, motivations, and past successes) and planning (change successfully) (Miller and Rollnick, 2013; Graves and Watkins, 2015). The aim of engaging is to encourage the individual to engage in the change process, take ownership of their difficulties and/or ambivalence and to identify their strengths. The aim of focusing is to elicit the client's agenda, point out possible choices, and ask permission to discuss your own agenda. Evoking is to reveal the individual's own motivation to change and planning encompasses commitment to change and formulation of a concrete plan of action (Miller and Rollnick, 2013). The core values of the method are that it is nonjudgmental, nonconfrontational, and nonadversarial (Miller and Rollnick, 2012). Motivation to change is directed by the client and not imposed by the counselor. The counselor's task is to be supportive and help the client to resolve ambivalence. Counseling skills and techniques that are highlighted within MI are often referred to as the "OARS" principles; Openended questions (encourage the clients to respond to questions from his/her own perspective, Affirmations (actively listening for the client's strengths and values, and reflecting these back in an affirming manner), Reflective listening (expressing empathy as well as guiding and supporting the client toward change), and Summary reflections (recaps and calls attention to important elements of the discussion).

The clinical relevance of MI has been empirically tested, and a positive effect of the technique has been demonstrated in $74 \%$ of randomized controlled trials (RCTs) (Rubak et al, 2005), although none of those studies specifically addressed hearing aid use. Psychologists and physicians have obtained a positive effect in approximately $80 \%$ of the studies, whereas other healthcare providers have obtained beneficial effects in $46 \%$ of the studies. Even brief encounters (15 min) have shown an effect (Rubak et al, 2005). MI has been evaluated in relation to weight loss (Tripp et al, 2011), alcohol, and drug abuse (D'Amico et al, 2015; Kohler and Hofmann, 2015; Korcha et al, 2015; Satre et al, 2016), eating disorders (Cassin et al, 2008; Weiss et al, 2013; Dray et al, 2014), pain (Miller-Matero and Cano, 2015), smoking cessation (Ha and Choi, 2012), and improved physical activity (Hardcastle et al, 2012).

MI relies on identifying and mobilizing the client's intrinsic values and goals to stimulate behavior change (Miller and Rollnick, 1991). The method is compatible with the four stages of change that hearing-impaired adults go through in audiological rehabilitation: precontemplation, contemplation, preparation, and action (Laplante-Lévesque et al, 2013). Reluctance to use one's hearing aid, whether because of no perceived need for it (Gussekloo et al, 2003; Lupsakko et al, 2005; Gopinath et al, 2011; Öberg et al, 2012), negative experiences with it, or unrealistic expectations regarding its usage (Hartley et al, 2010; Solheim, 2011; Öberg et al, 2012), are issues that can be explored in MI counseling. Such discussions can reveal preconceptions and objections to hearing aid use, as well as ambivalence about behavior change (Hickson et al, 2014; Meyer, Hickson, Khan, et al, 2014; Ridgway et al, 2015; Ferguson et al, 2016). Considering that motivation and self-efficacy are frequently reported factors found to be essential for hearing aid readiness and adoption, follow-up appointments should focus on issues that might affect the client's perceived benefit and satisfaction. Considering the four stages of change that hearing-impaired adults go through in audiological rehabilitation (Laplante-Lévesque et al, 2013), it may be helpful to determine the individual's stage of change to better tailor supportive services to their current needs. Nonetheless, it may be that low-users have different reasons for use and nonuse than more regular users, regardless of their stage of change, or that they progress through the stages of change in different ways than more regular users.

A recent RCT evaluated the use of MI to facilitate hearing aid use among low-users ( $\leq 4 \mathrm{~h} /$ day) among 37 older adults (mean age of $72 \mathrm{yrs}$ ) (Aazh, 2016a). All 37 patients were fitted with Oticon Zest hearing aids and participated in a 60 min hearing aid review, which consisted of: discussing any hearing aid problems, problem 
solving and practice, checking comfort and suitability of hearing aids, adjustment of hearing aid settings (if needed), and brief education about their hearing status, why they needed a hearing aid, how it works, and its limitations. Patients assigned to standard care (SC) received the hearing aid review only $(\mathrm{n}=17)$, whereas patients assigned to the motivational interviewing with standard care (MISC) group $(\mathrm{n}=20)$ received MI combined with SC. Mean pure-tone average audiometric threshold (PTA) for the better ear at the frequencies $0.25-4 \mathrm{~Hz}$ was similar for both the SC (30) and MISC (31) groups. The MI sessions were audio-recorded and transcribed, and coded using the MITI 3.1.1 behavioral coding system, a treatment integrity measure for clinical trials of motivational interviewing. Findings showed that, one month after the MI intervention, individuals who received MISC at the follow-up session used their hearing aids an average of $3.2 \mathrm{~h}$ more each day compared with individuals who only received SC. A qualitative study that explored patients' experiences taking part in a pilot study to evaluate the feasibility of a RCT of MI's effect on hearing aid use concluded that the three main themes that seemed to help people improve their hearing aid use were, a compassionate patient-clinician relationship, additional patient education and posthearing aid-fitting support (Aazh, 2016a). In addition to higher hearing aid use in the MISC group (Aazh, 2016a), the response to the optional follow-up in the MISC group was about $20 \%$ more than the SC group. The author suggests that the MI improved a patient's motivation to adhere to the study protocol, leading to a higher rate of acceptance of and attendance at the optional follow-up session (Aazh, 2016b).

There is limited knowledge and research on the effect of using MI with elderly hearing aid owners who rarely or never use their hearing aids. MI, which is designed to elicit, clarify, and resolve ambivalence, could identify and mobilize the client's goals regarding hearing aid use. The aim of this pilot study was to evaluate the feasibility and effects of providing a brief MI counseling session at a six-month follow-up appointment to elderly hearing aid recipients who rarely or never used their hearing aids in the prior six months (datalogged average usage of $<90 \mathrm{~min} /$ day). The effect of the counseling was evaluated in relation to an objective measure of hearing aid use (datalogging) three months later. A second aim was to describe clients' subjective assessments of their perceived need for hearing aids three months after MI counseling.

\section{METHODS}

\section{Design and Setting}

This study is part of a larger study of hearing aid use among elderly hearing aid recipients. The analysis described in this paper focuses on a subset of 47 participants who were provided with an MI counseling session six months after being fitted for a new hearing aid. Hearing aid use and experiences were assessed three months later, at a nine-month follow-up visit. The study did not include a control group. The study took place at Lovisenberg Diakonale Hospital, which serves as the only public Hearing Centre in Oslo, Norway. The average age of clients fitted for a hearing aid at the Hearing Centre is approximately 75 yrs.

\section{Ethics}

The study received approval from the National Committee for Research Ethics and the Norwegian Social Science Data Services and was conducted in 2013-14. All participants were provided both verbal and written information about the study, and all participants provided written informed consent.

\section{Participants}

All individuals fitted for a new hearing aid during the 12 months study period were invited to participate in the study. To be included in the larger study, potential participants needed to be $\geq 60$ yrs of age and able to communicate in Norwegian. Of the 256 clients invited to participate in the larger study at the time of their hearing aid fitting, 248 (97\%) consented, and 181 (73\%) attended the six-month follow-up appointment. Because data were not available for the 75 clients who did not return for the six-month follow-up, we could not determine whether they differed from those who did return with respect to hearing loss or hearing aid use. Average hearing loss in the participant's better ear $(0.125-8000 \mathrm{kHz}$ ) was $49.4 \mathrm{~dB}$ PTA (standard deviation $[\mathrm{SD}]=12.2$, range $23-92 \mathrm{~dB}$ PTA). All participants were fitted with a digital hearing aid with telecoils and a minimum of two listening programs. First-time hearing users as well as experienced users were included in the study. Participants were not excluded based on having other diseases or health conditions. Participants who attended the six-month follow-up visit and had objectively measured hearing aid use $<90 \mathrm{~min} /$ day were eligible to participate in the MI component of the study.

\section{Procedures}

\section{Hearing Aid Fitting}

Participants were referred to the Department of Otolaryngology at the Lovisenberg Diakonale Hospital for hearing aid fitting by an ear, nose and throat specialist. At the first appointment at the hospital, participants were examined by an ear, nose and throat specialist, then by an audiologist who performed hearing and speech discrimination testing, provided information on 
hearing aids, and assessed the individual's experience of hearing loss and perceived the need for hearing aid(s). Hearing aid fitting was performed according to the individual's hearing loss, preferences, available program settings, and previous experiences.

\section{Six-Month Follow-Up Appointment}

All individuals fitted for a hearing aid during the 12 months study period were advised to return for an optional six-month follow-up appointment. Detailed data regarding the site of lesion and etiology of hearing loss were not collected.

The six-month follow-up appointment included a 30 min session with a technical audiologist followed by a 30 min session with an educational audiologist. The session with the technical audiologist focused on hearing aid adjustments, practical issues associated with handling, and listening comfort, and registration of datalogged hearing aid use for the prior six months. The session with the educational audiologist focused on preconceptions/expectations, experiences, motivational factors, and self-assessments regarding the client's perceived benefit from and satisfaction with their hearing aid. Participants identified with objectively measured hearing aid use of $<90 \mathrm{~min} /$ day during the prior six months were provided with a $30 \mathrm{~min}$ MI counseling session.

\section{MI}

The MI approach was used to explore potential ambivalence to hearing aid use and to encourage participants with low use to increase their hearing aid use. Thus, only individuals with low use of hearing aids during the six months before the six-month follow-up visit were invited to participate in this part of the study. An educational audiologist educated and trained in the MI method conducted the MI counseling session at the end of the six-month follow-up visit. The educational audiologist had participated in a two-day workshop on MI, which included theory, skills, techniques, and practical issues. In addition, the audiologist was educated in cognitive therapy and had three years of experience with counseling techniques in clinical practice.

Each MI session was performed in accordance with the established principles of MI (Miller and Rollnick, 2013), and the aim was to discuss challenges to hearing aid use and find solutions acceptable to the client. The four stages of MI (i.e., engaging, focusing, evoking, and planning) were followed throughout the conversation, using the OARS skills previously described. Each interview began with the question: "Tell me how has it been with your hearing aids?" The participants were invited to recap their experiences related to hearing aid use for the last six months and to make their own assessments regarding benefit, comfort, and fitness. Because participants had different reasons for not using their hearing aids, we did not standardize a preset list of questions. Instead, a checklist of key points was used in exploring potential barriers, obstacles, and/or low motivation to hearing aid use. The conversation was based on the individual's experiences and opinions, and the checklist was used to categorize the types of problems and issues raised during the conversation and to form a basis for further focus and priorities held by the participant. As there is no standard tool for such assessment, we asked about nine specific issues known to be important in relation to hearing aid use. These were issues regarding handling, sound quality, perceived need, benefit, earmold/dome, economic factors, functional factors, cosmetic factors, health-related factors, and other. Potential problems and obstacles to hearing aid use were identified and discussed, and a solution-oriented approach was used to handle and solve potential barriers.

As the MI session emphasized engagement and commitment to hearing aid use, the interview focused on helping the client to recognize and do something about their current problems and/or reluctance about hearing aid use. In addition, the interview emphasized exploring positive aspects of behavioral change and potential benefits of using hearing aids. A typical question was What are some of the good things about using hearing aids? Follow-up questions addressed where and when hearing aid use could be beneficial and discussion of the potential benefits of hearing aid use for the particular event(s) mentioned by the participant.

The interviews were not recorded or observed; instead issues raised during the conversation were documented using the checklist, which served as a record of the participant's hearing aid experiences. At the end of the MI session, participants were informed that their hearing aid use and experiences would be assessed again three months later at a nine-month follow-up appointment. The participants were also informed that they would be offered $30 \mathrm{~min}$ with a technical audiologist at the nine-month follow-up appointment, if needed.

\section{Nine-Month Follow-Up Appointment}

Three months after the MI session, the participants returned for a nine-month follow-up appointment. The nine-month visit was conducted by an educational audiologist and was designed to last $30 \mathrm{~min}$. The visit focused on assessing the participant's hearing aid use and experiences for the prior three months. Advantages of and potential obstacles to hearing aid use were discussed, and their experiences were described and summarized. Further initiatives for promoting and facilitating hearing aid use were identified. If needed, consultation with 
a technical audiologist was also provided to address technical issues.

\section{Measures}

\section{Hearing Aid Use}

Hearing aid use was objectively assessed six and nine months after the hearing aid was fitted (i.e., before and three months after the MI session) using the hearing aid's digital datalogging feature. Because of the different types of datalogging systems (with or without decimals in the time-indicator), the frequency of use was rounded to the nearest hour (i.e., $<0.5 \mathrm{~h}=0 \mathrm{~h}, \geq 0.5 \mathrm{~h}=$ $1 \mathrm{~h}$ ). If the datalog differed between the participant's left and right hearing aids, the hearing aid with the higher average duration of use was used in the analysis.

\section{Hearing Aid Experiences}

At the nine-month follow-up appointment (three months after MI), participants were asked to describe their experiences with their hearing aid in the prior three months (since the six-month follow-up visit). Using open-ended questions delivered in a neutral tone, participants were asked about their experiences managing their hearing aid (e.g., inserting the earmold, changing the battery or filter, sound quality, and earmold fit and comfort), their perceived need for a hearing aid, as well as their perceived benefit from and contentment with using a hearing aid. Participants were also asked how their experiences may have changed since the six-month follow-up. The participants were given the opportunity to describe their experiences and perceptions in their own way. Based on the discussion, participant responses were coded on four dimensions: any management issues (yes or no), perceived need for a hearing aid (has need or has no need), change in perceived need for hearing aid since prior visit (increased or not), and increase in perceived benefit from or contentment with hearing aid (increased or not).

\section{Data Analysis}

Analyses were performed using SPSS 23.0 for Windows (IBM Corp., Armonk, NY). Descriptive statistics (means, SDs, and frequencies) were used to summarize sample characteristics. Independent $t$-tests were used to compare groups on continuous variables, separate variance $t$-tests with adjusted degrees of freedom were used for samples with unequal variances, and results were confirmed with Mann-Whitney $U$-tests to ensure that nonnormal distributions were not unduly influencing the findings. Because hearing aid use was not normally distributed (particularly at the six-month follow-up), the Wilcoxon signed-rank test was used to compare change in hearing aid use from the six-month to the ninemonth follow-up, and Spearman correlations were used to assess their degree of association. Spearman correlations were also used to assess associations between continuous participant characteristics and hearing aid use at nine months. A significance level of $p<0.05$ was used for all analyses.

\section{RESULTS}

$\mathrm{O}$ $\mathrm{f}$ the 181 participants who attended the six-month follow-up appointment, 47 (26\%) were categorized as hearing aid low-users (datalogged use $<90 \mathrm{~min} /$ day). All 47 low-users participated in the MI session and were included in this analysis. The 47 low-users were significantly younger than the 134 more frequent users $(76.8$ $[\mathrm{SD}=8.9]$ yrs versus $80.1[\mathrm{SD}=9.8]$ yrs, $t(179)=2.00$, $p=0.047)$, had less hearing loss $(44.2[\mathrm{SD}=8.8] \mathrm{dB}$ versus $51.1[\mathrm{SD}=12.7] \mathrm{dB}$, separate variance $t(116)=$ $4.10, p<0.001$ ), and were more likely to be first-time hearing aid users $\left(62 \%\right.$ versus $39 \%, \chi^{2}(1)=7.38, p=$ 0.007 ), but the 47 included and 134 excluded participants did not differ by gender (51\% versus $44 \%$ male, respectively, $\left.\chi^{2}(1)=0.69, p=0.405\right)$. The 47 included low-users were slightly less likely to have adjustments made to their hearing aids at the six-month visit, but the difference did not reach statistical significance (62\% versus $\left.76 \%, \chi^{2}(1)=3.60, p=0.058\right)$.

The sample characteristics for the 47 low-users included in the analysis are described in Table 1. Of the 47 low-users who received MI at the six-month follow-up appointment, 37 (79\%) returned for the ninemonth follow-up and 10 (21\%) did not. The 10 participants who did not attend the nine-month visit did not differ from the 37 who attended on any of the variables in Table 1, nor did they differ with respect to their hearing aid usage at the six-month visit $(0: 36$ [SD $=$ $0: 31]$ versus $0: 21$ [SD $=0: 29$ ], respectively, $t(45)=$ $1.42, p=0.162$ ).

Table 1. Characteristics of Nonusers and Low-Users Who Participated in the MI Session at the Six-Month Follow-Up Visit

\begin{tabular}{|c|c|c|}
\hline Characteristic & Mean (SD) & $\%(n)$ \\
\hline$\overline{\text { Age (yrs) }}$ & $76.8(8.9)$ & \\
\hline Gender, female & & $49 \%(23)$ \\
\hline Hearing loss (dB PTA $)^{a}$ & $44.2(8.8)$ & \\
\hline Prior hearing aid experience & & $38 \%(18)$ \\
\hline Years of experience ${ }^{\mathrm{b}}$ & $7.8(5.2)$ & \\
\hline Adjustments made at six months ${ }^{c}$ & & $62 \%(29)$ \\
\hline
\end{tabular}

Note: $\mathrm{N}=47$.

aln better ear.

${ }^{\mathrm{b}}$ For the 18 participants with prior experience.

${ }^{\mathrm{c}}$ Adjustments to hearing aid included adjustments of gain-frequency response, tests for occlusion effect and acoustic feedback, and modifications of acclimatization setting, programs, and loop system. 
As shown in Table 2, the 37 participants who attended the nine-month follow-up visit increased their datalogged hearing aid use from an average of 0:21 $(\mathrm{SD}=0: 29)$ to $1: 52(\mathrm{SD}=1: 40)$ (Wilcoxon $Z=4.61$, $p<0.001$ ). The Spearman correlation (rho) between hearing aid use at six and nine months was $0.34(p=0.040)$, indicating that longer use at six months was moderately associated with longer use at nine months. Moreover, 19 (51\%) of the 37 participants who attended the ninemonth visit increased their hearing aid use to an average of $2 \mathrm{~h} /$ day or more. However, it is unknown whether the 10 participants who did not return for the nine-month visit had any change in their hearing aid use after the MI and technical support visit.

Hearing aid use after MI did not differ for men (1:45 $[\mathrm{SD}=1: 20])$ and women $(2: 00$ [SD $=2: 02], t(35)=0.45$, $p=0.656$ ). Similarly, new hearing aid users had similar hearing aid use after MI (1:41 [SD = 1:42]) as participants with prior hearing aid experience $(2: 08$ [SD = $1: 39$ ], $t(35)=0.80, p=0.427)$. A comparison of participants who did and did not have adjustments made to their hearing aid at the six-month visit indicated no overall effect on hearing aid use at nine months $(2: 05$ [SD $=1: 56]$ versus 1:28 [SD $=0: 58]$, respectively; $t(35)=1.08, p=0.286)$. In addition, there were no significant correlations between hearing aid use after $\mathrm{MI}$ and the participant's age (rho $=0.15, p=0.364)$ or degree of hearing loss (rho $=-0.23, p=0.173$ ).

When asked about their hearing aid experiences in the three months after receiving $\mathrm{MI}$ and technical support, fewer than half $(41 \%)$ reported ongoing hearing aid management issues, and about a third (32\%) reported no perceived need for a hearing aid (Table 3). Although hearing aid management issues were not significantly associated with hearing aid use, participants who perceived no need for a hearing aid used their hearing aid, on average, for less than half the time of participants with perceived need for a hearing aid $(t(35)=2.57, p=0.014)$.

Table 2. Datalogged Hearing Aid Use at the Nine-Month Follow-Up Visit

\begin{tabular}{|c|c|c|}
\hline $\begin{array}{l}\text { Datalogged } \\
\text { Hearing Aid Use }\end{array}$ & $\begin{array}{l}\text { Six-Month Visit } \\
\text { (before MI) }\end{array}$ & $\begin{array}{c}\text { Nine-Month Visit } \\
\text { (after MI) }\end{array}$ \\
\hline Hours:minutes/day, mean (SD) & $0: 21(0: 29)$ & $1: 52(1: 40)$ \\
\hline \multicolumn{3}{|l|}{ Frequencies, \% (n) } \\
\hline 0 h/day & $60 \%(28)$ & $13 \%(6)$ \\
\hline 1 h/day & $40 \%(19)$ & $26 \%(12)$ \\
\hline 2 h/day & & $23 \%(11)$ \\
\hline 3 h/day & & $7 \%(3)$ \\
\hline 4 h/day & & $4 \%(2)$ \\
\hline 5 h/day & & $4 \%(2)$ \\
\hline 8 h/day & & $2 \%(1)$ \\
\hline Did not attend & & $21 \%(10)$ \\
\hline
\end{tabular}

When asked about changes during the prior three months, more than half of the 37 participants who attended the nine-month follow-up visit reported increased need for a hearing aid (59\%) and increased benefit and contentment with their hearing aid (57\%) (Table 3). Furthermore, these perceptions of increased need and benefit were also associated with increased hearing aid use, with mean use being an average of about two hours longer among those who perceived increased need or benefit.

\section{DISCUSSION}

ur findings provide evidence that a follow-up appointment with emphasis on motivational factors is effective for increasing hearing aid usage, even among clients identified as rarely or never using their hearing aid. However, it should be noted that these improvements were observed following a comprehensive follow-up appointment, which included both technical support and MI, and both are likely needed to address the varied issues that can limit hearing aid use. Previous studies have concluded that counseling is an important part of the fitting process and can have a significant effect on hearing aid use and satisfaction (Vuorialho et al, 2006; Laplante-Lévesque et al, 2013). Prefitting counseling has been found to have a small but significant effect on expectations, and having enough time for education, training and questions at the auditory center has been found to be an essential indicator for hearing aid use (Saunders et al, 2009; Solheim et al, 2012). Although previous studies have shown some benefit of counseling alone, we believe that combining it with MI, as demonstrated in the current study, can lead to even better results.

If motivation for using hearing aids is weak, potential technical problems may represent an additional barrier to hearing aid use and vice versa. Handling problems (Vuorialho et al, 2006; Öberg et al, 2012) and physical impairment (Solheim et al, 2011; Chen et al, 2014; Gispen et al, 2014) have also been shown to negatively impact motivation and mastery of hearing aids. Providing additional "refresher" information on hearing aid usage and handling and providing the opportunity to make hearing aid adjustments are likely key elements to giving low-users a "fresh start" with their hearing aid. Elderly individuals are often particularly vulnerable to technical and handling issues because of increasing health problems and cognitive impairment. Thus, follow-up appointments combining both technical support and MI counseling may be particularly helpful for elderly hearing aid users.

This study evaluated the impact of a single $30 \mathrm{~min}$ MI session at a follow-up appointment six months after a hearing aid fitting. This brief session might be considered insufficient to adequately explore motivational 
Table 3. Hearing Aid Use at the Nine-Month Follow-Up Visit Based on Reported Hearing Aid Experience

\begin{tabular}{|c|c|c|}
\hline Reported Hearing Aid Experience at Nine Months & $\%(n)(n=37)$ & Hearing Aid Use at Nine Months, Mean (SD) \\
\hline \multicolumn{3}{|l|}{ Hearing aid management issues ${ }^{a}$} \\
\hline No & $59 \%(22)$ & $2: 08(2: 00)$ \\
\hline Yes & $41 \%(15)$ & $1: 28(0: 55)$ \\
\hline \multicolumn{3}{|l|}{ Perceived need for hearing aid } \\
\hline Had no perceived need & $32 \%(12)$ & $0: 55(0: 48)^{b}$ \\
\hline Had perceived need & $68 \%(25)$ & $2: 19(1: 48)^{b}$ \\
\hline \multicolumn{3}{|c|}{ Change in perceived need for hearing aid since six-month visit } \\
\hline No increased need & $41 \%(15)$ & $0: 48(0: 47)^{\mathrm{C}}$ \\
\hline Increased need & $59 \%(22)$ & $2: 36(1: 44)^{c}$ \\
\hline \multicolumn{3}{|c|}{ Change in perceived benefit from or contentment with hearing aid since six-month visit } \\
\hline No increased benefit or contentment & $43 \%(16)$ & $0: 48(0: 45)^{\mathrm{c}}$ \\
\hline Increased benefit or contentment & $57 \%(21)$ & $2: 40(1: 45)^{\mathrm{c}}$ \\
\hline
\end{tabular}

issues associated with hearing aid use. However, the intent of the current study was to conduct a preliminary evaluation of a model that would pose minimal burden to elderly clients and could easily be implemented in hearing clinics, with minimal modification of current procedures and minimal additional resources. Thus, it is promising that such a brief intervention was effective for increasing use among clients who rarely or never use their hearing aid. Nonetheless, additional follow-up sessions may also be useful to address the wide range of technical and motivational issues that clients may face. Further research is needed to determine whether additional sessions would result in more frequent or more sustained usage and whether including MI as part of the initial fitting might lead to earlier adoption of hearing aid use.

In a prior study, Aazh (2016b) reported a larger increase in hearing aid use after MI than observed in the present study. That study reported a six-hour increase in usage (compared to a three-hour increase in the control group) after one month, whereas we observed a one to two hours increase in usage. These differing findings may be due to several methodological differences between the studies. First, the current study only included participants who used their hearing aid on an average of $<90 \mathrm{~min} /$ day, whereas Aazh's study included participants with higher levels of use ( $\leq 4 \mathrm{~h} /$ day). It may be easier to increase usage among clients who are already using their hearing aids on a somewhat regular basis than among clients with no consistent pattern of usage, and the barriers to usage likely differ for these groups. Second, in contrast to Aazh's study, the current study did not exclude participants for poor manual dexterity, which may have interfered with participants' ability to manage and use their hearing aids and would presumably be less responsive to $\mathrm{MI}$ or technical support. Third, the Aazh study evaluated the effect of MI after one month, whereas we evaluated the effects after three months. It is possible that the benefits of MI diminish over time, and the differing follow-up periods between the two studies might help explain our more modest findings.

We found that nearly half of the study participants experienced challenges managing their hearing aids three months after the six-month follow-up appointment (Table 3). Impaired health and age-related declines in sensory abilities may affect older individuals' physical and psychosocial function, as well as their ability to manage their hearing aids. Consequently, focus on hearing loss alone may not be sufficient for the treatment of elderly, hearing-impaired individuals. Previous studies have shown that hearing-impaired older adults report greater difficulties with functional activities and more comorbidities than older adults without hearing loss (Barrenäs and Holgers, 2000; Chen et al, 2014; Gispen et al, 2014). Such findings suggest that the total limitations experienced in everyday life may have an additive effect.

Nearly a third (32\%) of our participants indicated that they experienced no need for hearing aids at the nine-month follow-up appointment (three months after MI counseling), and not surprisingly, this subgroup had substantially lower hearing aid use than the rest of the sample. These results are consistent with previous findings that hearing impairment itself is less significant than perceived need as a determinant of hearing aid use and that self-assessments and functional effects of hearing loss in everyday life are what matters (Hickson and Worrall, 2003). Consequently, it must be accepted that some elderly adults with hearing 
impairment may choose not to use hearing aids after trying them. Nonetheless, when clients have been fitted with a hearing aid and are later identified as rarely or never using it, follow-up should be standard to explore potential problems and motivational issues and to determine whether further support is warranted.

\section{Limitations}

This pilot study had several limitations that need to be considered. The most significant limitation is that this preliminary study had no control group, and thus it cannot be ruled out that the participants would have increased their hearing aid use even without the MI counseling and technical support. Nonetheless, all other things being equal, past behavior is usually a good indicator of future behavior, and after six months of using a hearing aid rarely or not at all, spontaneous improvement would seem to be unlikely. In addition, the participants in this study were offered MI as well as technical support, which may have included hearing aid adjustments. Although the effect of a hearing aid adjustment was not statistically significant in this small sample, it cannot be ruled out that the hearing aid adjustments contributed to the observed increase in hearing aid use at the follow-up appointment. Because MI counseling was provided in conjunction with technical support, we were unable to determine the individual contributions of each component to the observed findings. However, in light of the promising findings, RCTs are warranted to determine the appropriate target group and intervention dose and content to achieve optimal results. Moreover, MI might also be evaluated for its effectiveness when implemented at the initial hearing aid fitting rather than waiting until clients are found to have low use.

Although it was a strength that the study included an objective measure of hearing aid use, the datalog provided a crude measure of usage (average hours per day), and due to differences in the level of precision across datalogs, for consistency, all values were rounded to a whole hour. As a result, our estimates of usage have limited precision and do not reflect shifts in usage that may have occurred across the follow-up periods. More precise datalogging would strengthen future studies. Moreover, the increase in datalogged hearing use observed in this study was modest, and consideration should be given to how this increase might be further improved.

The aim of MI is to enhance motivation for behavior change, yet changes in motivation were not specifically measured in this study. Increased motivation might be inferred from the observed increases in hearing aid use, but several other variables could also explain these results, including adjusted expectations, a stronger sense of confidence or self-efficacy because of learning new information and skills, or a positive response to the general personal support provided during the MI session. Nonetheless, increases in datalogged hearing aid use suggest that the participants may have learned new behavioral skills, which is also a key goal of MI.

In the current pilot study, each MI session was performed according to the established principles of MI (Miller and Rollnick, 2013). Although our findings are promising, future studies would be strengthened by recording, transcribing, and coding the interviews using the MITI 3.1.1. behavioral coding system. In addition, participants in the current study used different types of hearing aids according to their preference, and it cannot be ruled out that this may have influenced the observed findings. Future studies would be strengthened by either standardizing the type of hearing aid used by participants or statistically evaluating the potential effect of different hearing aids. Lastly, $21 \%(\mathrm{n}=10)$ of the participants who received the MI counseling in this study did not return for the follow-up appointment three months later, and it is unknown what impact the MI had on these participants. Comparisons between those who did and did not attend the nine-month followup appointment did not indicate any differences, and the reasons they did not attend are unknown.

\section{CONCLUSION}

I $\mathrm{n}$ this study, MI was used to help low-users of hearing aids increase their use by identifying reasons they might want to engage in behavior change. We found a significant increase in hearing aid use among low-users after a 30 min MI counseling session combined with technical support provided six months after the hearing aids were fitted. These findings indicate that followup appointments using MI counseling may be useful to increase hearing aid use among elderly adults who rarely or never use their hearing aids.

Acknowledgments. We thank ExtraStiftelsen and Lovisenberg Diakonale Hospital for facilitating the study.

\section{REFERENCES}

Aazh H. (2016a) Patients' experience of motivational interviewing for hearing aid use: a qualitative study embedded within a pilot randomised controlled trial. $J$ Phonet and Audiol 2:1-13.

Aazh H. (2016b) Feasibility of conducting a randomized controlled trial to evaluate the effect of motivational interviewing on hearingaid use. Int $J$ Audiol 55(3):149-156.

Barrenäs ML, Holgers KM. (2000) A clinical evaluation of the hearing disability and handicap scale in men with noise induced hearing loss. Noise Health 2(6):67-78.

Cassin SE, von Ranson KM, Heng K, Brar J, Wojtowicz AE. (2008) Adapted motivational interviewing for women with binge eating 
disorder: a randomized controlled trial. Psychol Addict Behav 22(3):417-425.

Chang YS, Choi J, Moon IJ, Hong SH, Chung WH, Cho YS. (2016) Factors associated with self-reported outcome in adaptation of hearing aid. Acta Otolaryngol 136(9):905-911.

Chen DS, Genther DJ, Betz J, Lin FR. (2014) Association between hearing impairment and self-reported difficulty in physical functioning. J Am Geriatr Soc 62(5):850-856.

Cherko M, Hickson L, Bhutta M. (2016) Auditory deprivation and health in the elderly. Maturitas 88:52-57.

D’Amico EJ, Houck JM, Hunter SB, Miles JN, Osilla KC, Ewing BA. (2015) Group motivational interviewing for adolescents: change talk and alcohol and marijuana outcomes. $J$ Consult Clin Psychol 83(1):68-80.

Dray J, Gilchrist P, Singh D, Cheesman G, Wade TD. (2014) Training mental health nurses to provide motivational interviewing on an inpatient eating disorder unit. J Psychiatr Ment Health Nurs 21(7):652-657.

Ferguson MA, Woolley A, Munro KJ. (2016) The impact of selfefficacy, expectations, and readiness on hearing aid outcomes. Int J Audiol 55(Suppl 3):S34-S41.

Gianopoulos I, Stephens D, Davis A. (2002) Follow up of people fitted with hearing aids after adult hearing screening: the need for support after fitting. BMJ 325:471.

Gimsing S. (2008) Use of hearing aids five years after issue. Ugeskr Laeger 170(43):3407-3411.

Gispen FE, Chen DS, Genther DJ, Lin FR. (2014) Association between hearing impairment and lower levels of physical activity in older adults. J Am Geriatr Soc 62(8):1427-1433.

Gopinath B, Schneider J, Hartley D, Teber E, McMahon CM, Leeder SR, Mitchell P. (2011) Incidence and predictors of hearing aid use and ownership among older adults with hearing loss. Ann Epidemiol 21(7):497-506.

Graves E, Watkins RW. (2015) Motivational interviewing: patient engagement as the key to healthy practices, patients, and practitioners. N C Med J 76(3):175-176.

Gussekloo J, de Bont LE, von Faber M, Eekhof JA, de Laat JA, Hulshof JH, van Dongen E, Westendorp RG. (2003) Auditory rehabilitation of older people from the general population-the Leiden 85-plus study. Br J Gen Pract 53(492):536-540.

Ha YS, Choi YH. (2012) Effectiveness of a motivational interviewing smoking cessation program on cessation change in adolescents. J Korean Acad Nurs 42(1):19-27.

Hardcastle S, Blake N, Hagger MS. (2012) The effectiveness of a motivational interviewing primary-care based intervention on physical activity and predictors of change in a disadvantaged community. J Behav Med 35(3):318-333.

Hartley D, Rochtchina E, Newall P, Golding M, Mitchell P. (2010) Use of hearing AIDS and assistive listening devices in an older Australian population. J Am Acad Audiol 21(10):642-653.

Hickson L, Meyer C, Lovelock K, Lampert M, Khan A. (2014) Factors associated with success with hearing aids in older adults. Int $J$ Audiol 53(Suppl 1):S18-S27.

Hickson L, Worrall L. (2003) Beyond hearing aid fitting: improving communication for older adults. Int $J$ Audiol 42(Suppl 2): 2S84-2S91.
Kaplan-Neeman R, Muchnik C, Hildesheimer M, Henkin Y. (2012) Hearing aid satisfaction and use in the advanced digital era. $\mathrm{La}$ ryngoscope 122(9):2029-2036.

Kohler S, Hofmann A. (2015) Can motivational interviewing in emergency care reduce alcohol consumption in young people? A systematic review and meta-analysis. Alcohol Alcohol 50(2):107-117.

Korcha RA, Polcin DL, Evans K, Bond JC, Galloway GP. (2015) Intensive motivational interviewing for women with alcohol problems. Counselor (Deerfield Beach) 16(3):62-69.

Laplante-Lévesque A, Hickson L, Worrall L. (2013) Stages of change in adults with acquired hearing impairment seeking help for the first time: application of the transtheoretical model in audiologic rehabilitation. Ear Hear 34(4):447-457.

Lupsakko TA, Kautiainen HJ, Sulkava R. (2005) The non-use of hearing aids in people aged 75 years and over in the city of Kuopio in Finland. Eur Arch Otorhinolaryngol 262(3):165-169.

Maeda Y, Sugaya A, Nagayasu R, Nakagawa A, Nishizaki K. (2016) Subjective hearing-related quality-of-life is a major factor in the decision to continue using hearing aids among older persons. Acta Otolaryngol 136(9):919-922.

Meyer C, Hickson L, Khan A, Walker D. (2014) What is important for hearing aid satisfaction? Application of the expectancydisconfirmation model. J Am Acad Audiol 25(7):644-655.

Meyer C, Hickson L, Lovelock K, Lampert M, Khan A. (2014) An investigation of factors that influence help-seeking for hearing impairment in older adults. Int J Audiol 53(Suppl 1):S3-S17.

Miller WR, Rollnick S. (1991) Motivational Interviewing: Preparing People to Change Addictive Behaviour. New York, NY: Guilford Press.

Miller WR, Rollnick S. (2012) Motivational Interviewing: Helping People Change. United Kingdom: Guilford Press.

Miller WR, Rollnick S. (2013) Motivational Interviewing: Helping People Change. 3rd ed. New York, NY: Guilford Press.

Miller-Matero LR, Cano A. (2015) Encouraging couples to change: a motivational assessment to promote well-being in people with chronic pain and their partners. Pain Med 16(2):348-355.

Öberg M, Marcusson J, Nägga K, Wressle E. (2012) Hearing difficulties, uptake, and outcomes of hearing aids in people 85 years of age. Int $J$ Audiol 51(2):108-115.

Ridgway J, Hickson L, Lind C. (2015) Autonomous motivation is associated with hearing aid adoption. Int J Audiol 54(7):476-484.

Rubak S, Sandbaek A, Lauritzen T, Christensen B. (2005) Motivational interviewing: a systematic review and meta-analysis. $\mathrm{Br} J$ Gen Pract 55(513):305-312.

Satre DD, Leibowitz A, Sterling SA, Lu Y, Travis A, Weisner C. (2016) A randomized clinical trial of motivational interviewing to reduce alcohol and drug use among patients with depression. J Consult Clin Psychol 84(7):571-579.

Saunders GH, Lewis MS, Forsline A. (2009) Expectations, prefitting counseling, and hearing aid outcome. J Am Acad Audiol 20(5): $320-334$.

Solheim J. (2011) Preconceptions and expectations of older adults about getting hearing aids. J Multidiscip Healthc 4:1-8.

Solheim J, Kværner KJ, Falkenberg ES. (2011) Daily life consequences of hearing loss in the elderly. Disabil Rehabil 33(23-24): 2179-2185. 
Solheim J, Kvaerner KJ, Sandvik L, Falkenberg ES. (2012) Factors affecting older adults' hearing-aid use. Scand $J$ Disabil Res 14:300-312.

Stark P, Hickson L. (2004) Outcomes of hearing aid fitting for older people with hearing impairment and their significant others. Int $J$ Audiol 43(7):390-398.

Tripp SB, Perry JT, Romney S, Blood-Siegfried J. (2011) Providers as weight coaches: using practice guides and motivational inter- view to treat obesity in the pediatric office. $J$ Pediatr Nurs 26(5):474-479.

Vuorialho A, Sorri M, Nuojua I, Muhli A. (2006) Changes in hearing aid use over the past 20 years. Eur Arch Otorhinolaryngol 263(4):355-360.

Weiss CV, Mills JS, Westra HA, Carter JC. (2013) A preliminary study of motivational interviewing as a prelude to intensive treatment for an eating disorder. J Eat Disord 1:34. 